\title{
Clonal reproduction by males and females in the little fire ant
}

\author{
Denis Fournier ${ }^{1 \star} \dagger$, Arnaud Estoup ${ }^{1 \star}$, Jérôme Orivel $^{2}$, Julien Foucaud ${ }^{1}$, Hervé Jourdan ${ }^{3}$, Julien Le Breton ${ }^{4}$ \\ $\&$ Laurent Keller ${ }^{5}$
}

Sexual reproduction can lead to major conflicts between sexes and within genomes ${ }^{1-4}$. Here we report an extreme case of such conflicts in the little fire ant Wasmannia auropunctata. We found that sterile workers are produced by normal sexual reproduction, whereas daughter queens are invariably clonally produced. Because males usually develop from unfertilized maternal eggs in ants and other haplodiploid species, they normally achieve direct fitness only through diploid female offspring. Hence, although the clonal production of queens increases the queen's relatedness to reproductive daughters, it potentially reduces male reproductive success to zero. In an apparent response to this conflict between sexes, genetic analyses reveal that males reproduce clonally, most likely by eliminating the maternal half of the genome in diploid eggs. As a result, all sons have nuclear genomes identical to those of their father. The obligate clonal production of males and queens from individuals of the same sex effectively results in a complete separation of the male and female gene pools. These findings show that the haplodiploid sex-determination system provides grounds for the evolution of extraordinary genetic systems and new types of sexual conflict.
The little fire ant has been introduced from neotropical lowland forests into North America, West Africa, Melanesia, Polynesia, the Galapagos and some subtropical Atlantic Islands, where it has become a major pest. Colonies consist of several spatially separated nests headed by multiple reproductive queen ${ }^{5}$. Although queens can participate in mating flights ${ }^{6}$, colonies spread largely or entirely by budding, a process in which one or more queens initiate a new colony in the vicinity of the mother nest with the help of workers ${ }^{5}$. While conducting a genetic population study of this species, we discovered a new genetic system in which females and males both reproduce clonally.

We collected 34 nests of $W$. auropunctata from five sites in French Guiana, which is within the native range (Fig. 1). The number of queens per nest was $4.2 \pm 0.7$ (mean \pm s.e.m.; range $0-18$ ). We genotyped at 11 highly polymorphic microsatellite loci (observed heterozygosities: $0.502-0.964)$ all the queens $(n=142)$ collected, the sperm in their spermathecae, the nine young winged queens (gynes) found in one of the 34 nests, and 264 workers $(7.8 \pm 0.2$ workers per nest). An analysis of these genotypes revealed a very unusual pattern that could be explained only by queens being produced by clonal
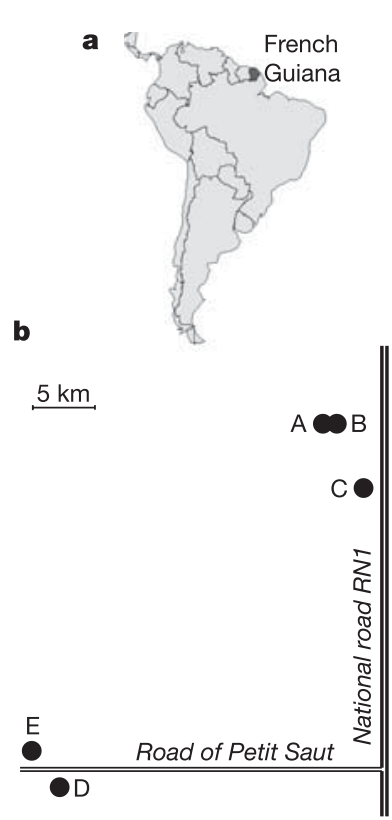

c
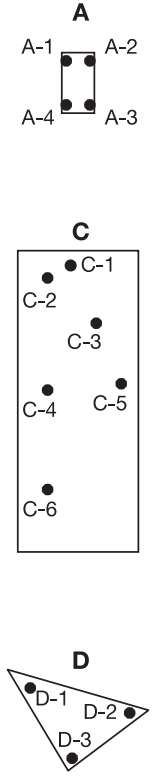

B

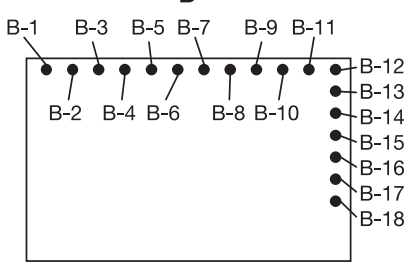

E

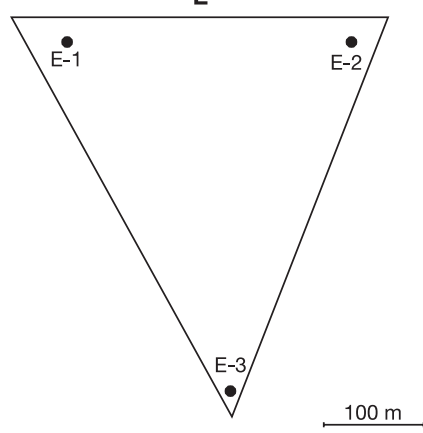

Figure $1 \mid$ Site and nest locations. a, b, Locations of the five sites of collection (A-E): national (a) and local (b). c, Locations of the nests within each of the five sites; scales are identical for each of the five sites.

${ }^{1}$ Centre de Biologie et de Gestion des Populations, INRA, Campus International de Baillarguet, CS 30 016, 34988 Montferrier/Lez Cedex, France. ${ }^{2}$ Laboratoire Évolution et Diversité Biologique, UMR-CNRS 5174, Université Toulouse III, 118 route de Narbonne, 31062 Toulouse cedex 4, France. ${ }^{3}$ Laboratoire de Zoologie Appliquée, IRD, 98948 Nouméa, Nouvelle-Calédonie. ${ }^{4}$ Laboratory of Sub-Tropical Zoology, University of the Ryukyus, 903-0213 Nishihara, Okinawa, Japan. ${ }^{5}$ Department of Ecology and Evolution, Bâtiment de Biologie, University of Lausanne, 1015 Lausanne, Switzerland. †Present address: Behavioral and Evolutionary Ecology - CP 160/12, Université Libre de Bruxelles, 50 avenue F. D. Roosevelt, 1050 Brussels, Belgium.

*These authors contributed equally to this work. 
Table 1 | Genotypes of queens (Q), their mates (M) and workers (W) in one nest (E-3) at each of the 11 microsatellite loci

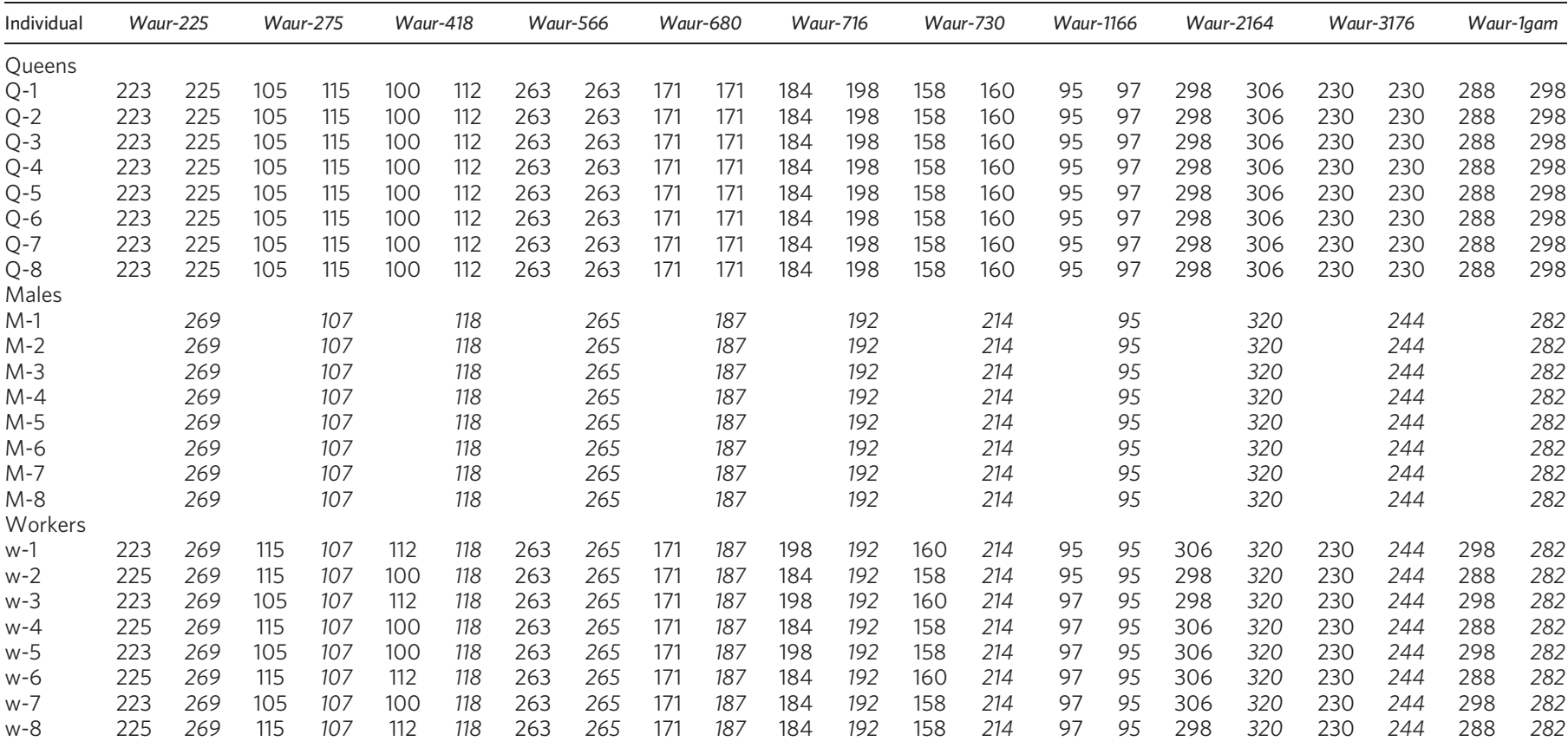

The identities of mates were determined by the sperm collected in the queen's spermathecae. Queens and males' genotypes illustrate their clonal production, whereas workers' genotypes are consistent with normal sexual reproduction. Paternal alleles are in italics.

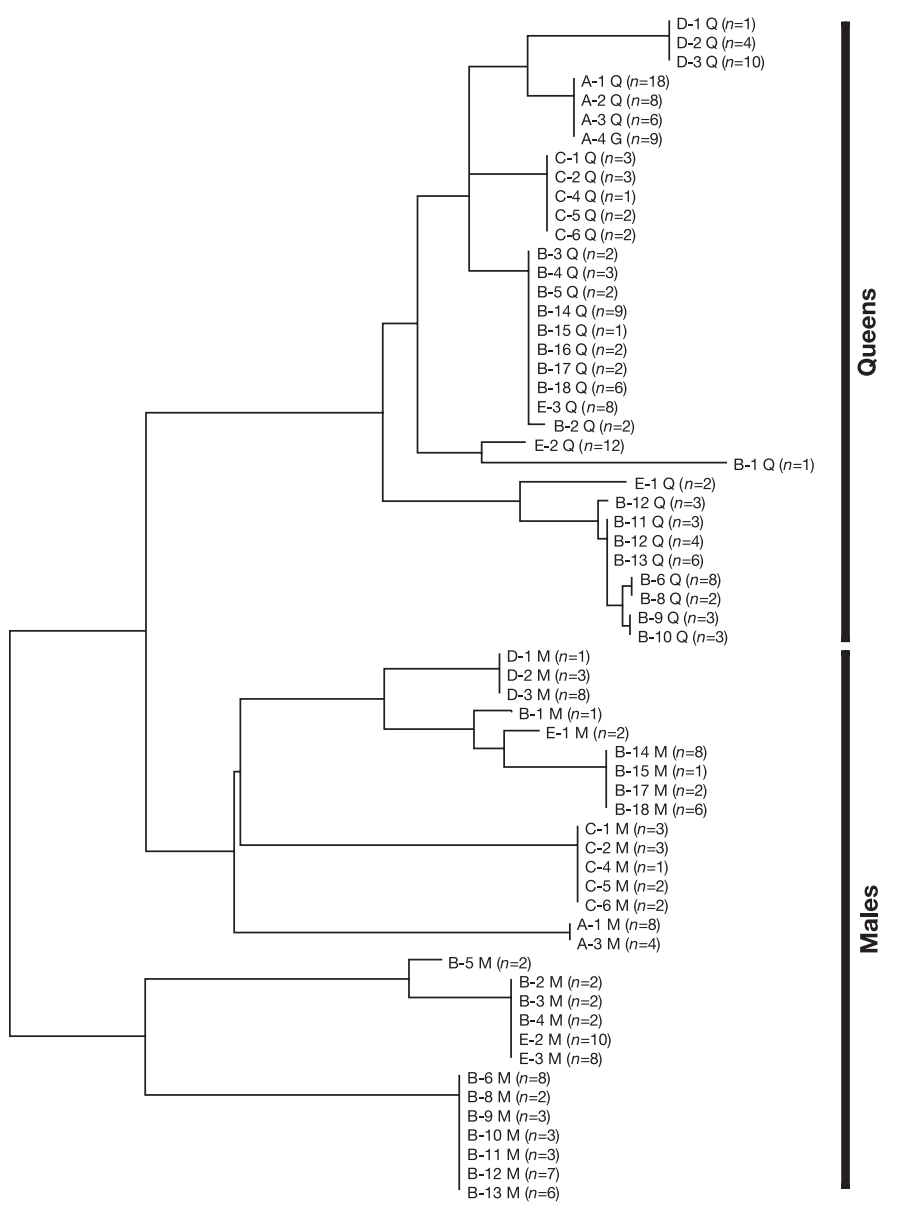

Figure 2 | Neighbour-joining dendrogram of the genetic (allele-shared) distances between queens $(Q)$, gynes $(G)$ and male sperms $(M)$ collected over all the five sites (A-E). The collection number of each nest is given with the letter of the site (see Fig. 1 for details). The number of individuals sharing the same genotype $(n)$ is given for all nests. reproduction (that is, by ameiotic parthenogenesis). In 33 of the 34 nests, all queens $(n=135)$ and gynes $(n=9)$ cohabiting in the same nest shared an identical genotype at each of the 11 loci (Table 1 and Fig. 1). The single exception was nest B-12, in which queens differed at 1 of the 11 loci: four queens were heterozygous at Waur-2164 and the remaining three queens were homozygous for one of the two alleles. This variation probably reflects a mutation or recombination event in one queen followed by clonal reproduction within the nest. The history of this genetic change could be reconstructed from the genotypes of queens collected in neighbouring nests (Figs 1 and 2). Nine queens from two neighbouring nests (B-11 and B-13) had the same genotype as the four heterozygous queens for locus Waur-2164, indicating that the mutation or recombination event probably was from a heterozygote to a homozygote queen. The three homozygote queens from nest B-12 had a unique genotype in the population, which further supports this interpretation.

A comparison between nests supports the view of restricted female gene flow, with budding being the main mode of colony formation. Within three of the five sites of collection (A, C and D) all queens had the same genotype at the 11 loci (Fig. 2). In one of the two other sites (B), all queens from 8 of the 17 nests also had an identical genotype, whereas in the other site (E) the queen genotypes were different in the three nests sampled. Taken together, these data indicate that queens belonging to the same lineage of clonally produced individuals frequently head closely located nests. Moreover, genetic differentiation between sites was very strong, with a single occurrence of genotypes shared between sites (the eight queens of nest E-3 had genotypes identical to the most common genotype found at site B), showing that gene flow by females is extremely restricted.

In stark contrast to reproductive females, the genotypic analyses revealed that workers are produced by normal sexual reproduction (Table 1). Over all 31 queenright nests, each of the 248 genotyped workers had, at seven or more loci, one allele that was absent in queens of their nest. Moreover, the 232 workers from the 29 nests in which the sperm in the queen's spermathecae was successfully obtained had all genotypes consistent with those expected under sexual reproduction between the two parental genomes.

The genetic analyses of the sperm collected in the queens' 
spermathecae revealed a pattern of partitioning of genetic variation that was remarkably similar to the one found in reproductive females. Cohabiting queens from a given nest were always inseminated by males having an identical genotype at the 11 loci (Table 1). Moreover, a single male genotype was found in the three sites (A, C and D) harbouring a single queen genotype. In contrast, the two remaining sites ( $\mathrm{B}$ and $\mathrm{E}$ ) that contained several queen genotypes also had several male genotypes. The observation that nests never contained more than one male genotype also supports the view that male dispersal is also extremely limited, with most or all matings taking place within the parental nest. This breeding system would account for nests invariably containing a unique queen and male genotype.

The finding that the males that inseminated queens of a given nest invariably had the same genotype, together with the fact that males and queens never have the same genotype, reveals that the genomes of males are also transmitted clonally. Additional evidence that males are indeed clonally produced from the sperm in the queens' spermathecae came from the genotypes of 41 males found in four of the nests (mean \pm s.e.m. $10.3 \pm 2.5$ ) collected in another population in New Caledonia. In each of the four colonies, males had genotypes (11 microsatellites) identical to those of the sperm found in the spermathecae of queens heading their colony (the $12.0 \pm 1.5$ queens per colony again had all the same genotype) and genotypes incompatible with maternal inheritance of their genomes (all males had alleles that were absent in queens at 10 of the 11 loci). In addition,
25 of the 41 males were pupae, indicating that they had been produced within their parental colony. The most likely mechanism for this mode of clonal reproduction is the paternal elimination of the maternal genome in the egg. Accordingly, the resulting haploid males produced would lack maternal genes and would have a genotype identical to the sperm stored in the queens' spermathecae. The alternative mechanism of androgenesis (the fusion of two sperm nuclei, leading to all-paternal diploid males) can be ruled out because flow cytometry analysis conducted on 10 male heads revealed that they were all haploid (S. Aron, personal communication).

By using alternative modes of reproduction for the queen and worker castes, queens can increase the transmission rate of their genes to their reproductive female offspring while maintaining genetic diversity in the worker force. The fact that sexual reproduction has been retained to produce workers indicates that sexual reproduction might have important benefits for colony function, for example through increased defence against parasites, more efficient division of labour and an increased range of environmental conditions that a colony can tolerate ${ }^{7-9}$. Thus, if queens were also to produce workers clonally, all females within colonies would have identical genomes, whereas the mode of reproduction that we have uncovered effectively leads to levels of colony genetic diversity identical to that expected in a typical colony headed by one queen mated with one male. A similar situation of conditional use of sex by queens has been reported in another ant, Cataglyphis cursor ${ }^{10}$. Interestingly, in C. cursor and W. auropunctata the cost of asexual
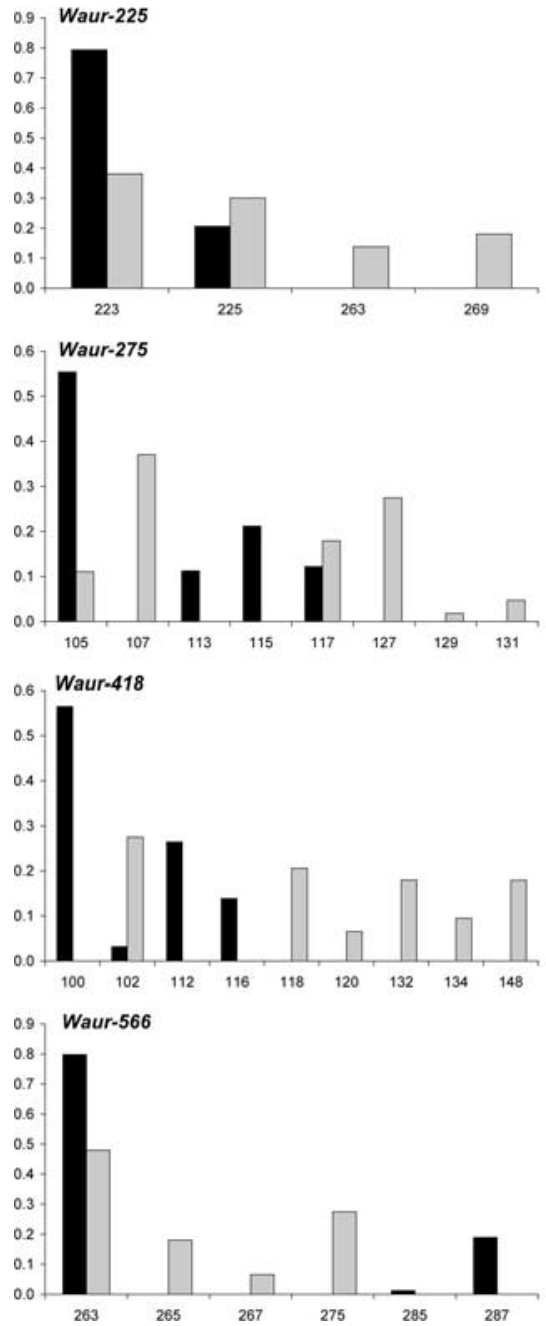
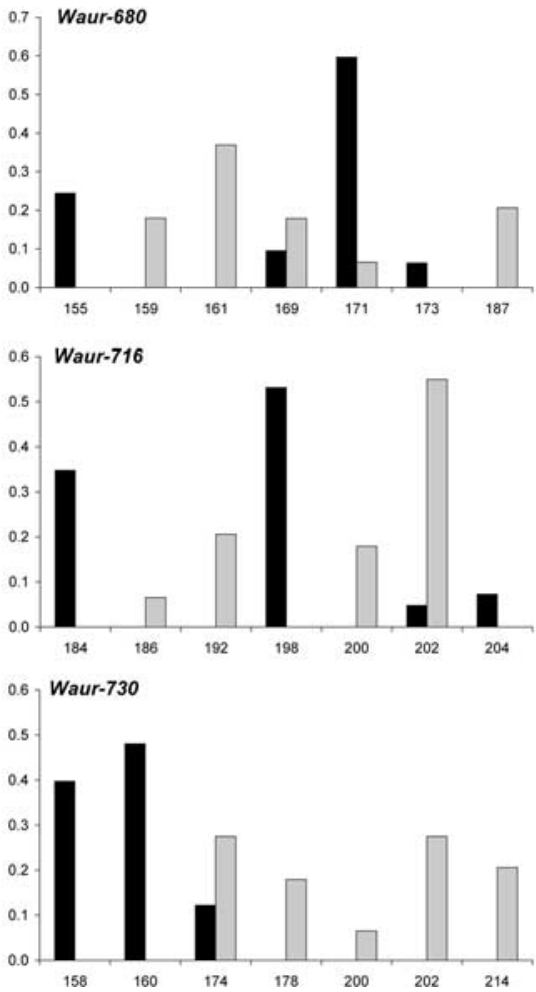
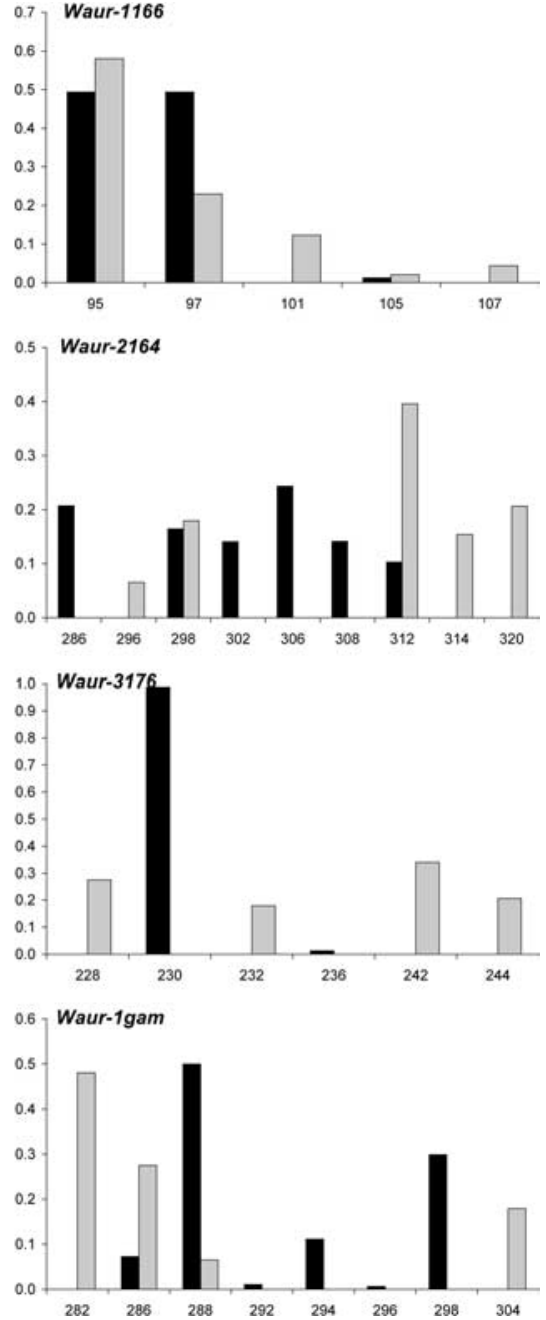

Figure 3 | Allele frequencies for queens (black bars) and their mates (grey bars) at each of the 11 microsatellite loci. 
reproduction might be lower than in most other ants because in these two species queens do not go through a stage of independent colony founding in which queens initiate a new colony without the help and protection of workers ${ }^{10}$. However, an important difference is that parent-offspring analyses revealed numerous cases of crossing-over events in C. cursor as a result of automictic parthenogenesis with central fusion, a process in which two of the four products of meiosis merge. In contrast, parthenogenesis seems to be only, or at least mostly, ameiotic in $W$. auropunctata as indicated by the fact that the vast majority of queens from the same nest and sometimes all the queens from the same site have the same genotype. The relatively high level of queen heterozygosity (mean \pm s.e.m. $0.619 \pm 0.028$ ) also shows a very low rate or the complete absence of recombination, because crossing-over should result in offspring becoming homozygous for one of the two maternal alleles and a rapid decrease in heterozygosity ${ }^{11}$.

To our knowledge there has been only one other report of clonal reproduction by males in the animal kingdom, in which it was found that 3 of 61 queens homozygous for a recessive (cordovan) mutation inducing a brown instead of the wild-type black colour produced some brown males ${ }^{12}$. This showed convincingly that some males could not originate from unfertilized eggs produced by queens, but it is possible that these males were diploid, as sometimes occurs in Hymenoptera ${ }^{13}$. Some support for the occurrence of diploid males comes from the observation that the three queens also produced some gynandromorphic males with parts of cordovan (brown) and dark coloured tissue, a pattern best explained by diploid males experiencing partial reduction of ploidy level during development. Although the available data do not allow us to conclude whether clonal reproduction by males does really occur in honeybees, it is important to note that this mode of reproduction might have remained unnoticed in social Hymenoptera because of the paucity of suitable parent-offspring genetic analyses. Good candidates for male clonal reproduction are species in which the reproduction of workers has been inferred on the basis that males harboured alleles absent in queens but present in their mates (and thus in workers).

The elimination of one of the two parental genomes during meiosis has been described in fishes, amphibians and several insect species $^{14,15}$. However, these cases invariably involve the elimination of the paternal genome. A case of male induction by genome elimination has also been reported in a haplodiploid hymenopteran, the wasp Nasonia vitripennis, in which a paternal-sex-ratio ( $p s r)$ chromosome induces the supercondensation and destruction of the paternal chromosomes (except psr) in early fertilized eggs ${ }^{16,17}$. The selfish effect of this B chromosome is to convert diploid eggs, which would have normally developed into females, into haploid males. However, in this system it is also the paternal and not the maternal genome that is eliminated. Thus, the $W$. auropunctata system described here is unique in that clonal reproduction occurs by the transmission of the paternal and not the maternal genome. This system might have evolved in response to the obligate clonal production of queens as a mechanism for males to achieve some reproductive success.

The occurrence of clonal reproduction by both males and queens has important consequences for the apportionment of genetic variability and genome evolution. Because genes are transmitted only between individuals of the same sex, there is effectively no gene flow between the male and queen gene pools. Genetic differentiation between the male and queen genomes can thus persist and accumulate, as demonstrated by the fact that the genomic compositions of queens differed notably from those of males (Fig. 3). Allele frequencies were significantly different between sexes (Fisher's exact tests, $P<0.00001$ for each of the 11 loci), with some loci such as Waur-3176 being diagnostic. A dendrogram analysis also revealed that males and queens clustered on separate branches of the tree (Fig. 2). There was a complete segregation of the queens' and the males' genomes. Accordingly, the heterozygosity of the sexually produced workers (mean \pm s.e.m. $0.858 \pm 0.013$; range $0.693-$ 0.989 ) was significantly higher than that of queens (permutation test, $P=10^{-5}$ ), and their genotypes showed a significant departure from Hardy-Weinberg equilibrium, with a strong excess of heterozygous genotypes at all loci ( $P<10^{-5}$ at each locus).

This study shows that, in the evolutionary battle of opposing sexes, $W$. auropunctata has evolved an unusual mode of reproduction with queens circumventing the twofold cost of sexual reproduction by transferring all their genes to the reproductive females while males thwart queens by also clonally transmitting their genomes to sons. Although the male and female genomes come together in workers, this does not translate into any genetic exchange because workers are completely sterile ${ }^{18}$. As a result, the male and female genomes are completely segregated and form two distinct genetic lineages. These findings show that haplodiploidy and the caste-determination system provide grounds for the evolution of extraordinary genetic systems and that sexual conflicts are central in evolution with the potential for shaping various interactions between the sexes and their gametes $^{19-23}$.

\section{METHODS}

Sampling. Queens, gynes (winged queens), males and workers of W. auropunctata were sampled in March 2004 within their native range, in French Guiana. Thirtyfour nests were surveyed and collected at five sites (Fig. 1): two coffee-tree plantations (A, $05^{\circ} 17^{\prime} 20.82^{\prime \prime} \mathrm{N}, 52^{\circ} 55^{\prime} 11.40^{\prime \prime} \mathrm{W} ; \mathrm{B}, 05^{\circ} 17^{\prime} 16.02^{\prime \prime} \mathrm{N}$, $\left.52^{\circ} 55^{\prime} 04.08^{\prime \prime} \mathrm{W}\right)$, a sand-pit (C, $\left.05^{\circ} 16^{\prime} 13.92^{\prime \prime} \mathrm{N}, 52^{\circ} 55^{\prime} 02.70^{\prime \prime} \mathrm{W}\right)$, an old encampment ( $\left.D, 05^{\circ} 04^{\prime} 21.18^{\prime \prime} \mathrm{N}, 52^{\circ} 01^{\prime} 47.16^{\prime \prime} \mathrm{W}\right)$ and a quarry $\left(\mathrm{E}, 05^{\circ} 04^{\prime} 16.81^{\prime \prime} \mathrm{N}, 52^{\circ} 02^{\prime} 44.70^{\prime \prime} \mathrm{W}\right)$. In addition, a sample of queens and males collected in New Caledonia along a forest road $\left(20^{\circ} 33^{\prime} 16.71^{\prime \prime} \mathrm{S}\right.$, $\left.164^{\circ} 47^{\prime} 53.23^{\prime \prime} \mathrm{E}\right)$, in a coffee-tree plantation $\left(22^{\circ} 01^{\prime} 57.05^{\prime \prime} \mathrm{S}, 166^{\circ} 16^{\prime} 12.09^{\prime \prime}\right.$ E) and in a rainforest $\left(22^{\circ} 10^{\prime} 18.13^{\prime \prime} \mathrm{S}, 166^{\circ} 45^{\prime} 37.16^{\prime \prime} \mathrm{E}\right)$ were included in this study.

Nuclear genotyping. To isolate sperm DNA, the queen's abdomen was dissected as described in ref. 24. Whole individual ants were ground in cetyltrimethylammonium bromide (CTAB) solution. DNA was extracted and purified in accordance with standard CTAB-based protocols. Queens, males, gynes, workers and seminal fluid were genotyped at 11 highly polymorphic microsatellite loci (Waur-225, Waur-275, Waur-418, Waur-566, Waur-680, Waur-716, Waur-730, Waur-1166, Waur-2164, Waur-3176 and Waur-1gam; see detailed protocol in ref. 25.).

Microsatellite analyses. Tests for departure from Hardy-Weinberg equilibrium and genic differentiation between male and female gene pools were assessed with the exact probability tests implemented in GENEPOP 3.2a (ref. 26). Pairwise individual allele-shared distances were estimated over all loci as

$$
\mathrm{ASD}_{\mathrm{XY}}=\frac{1}{L} \sum_{l=1}^{L} 1-\left[\left(n_{l \mathrm{X} \rightarrow \mathrm{Y}}+n_{l \mathrm{Y} \rightarrow \mathrm{X}}\right) /\left(G_{\mathrm{X}}+G_{\mathrm{Y}}\right)\right]
$$

where $L$ is the number of genotyped loci, $G_{\mathrm{X}}$ and $G_{\mathrm{Y}}$ are the numbers of gene copies in individuals $\mathrm{X}$ and Y $(G=2$ for diploid genomes and $G=1$ for haploid genomes), respectively, and $n_{I \mathrm{X} \rightarrow \mathrm{Y}}\left(n_{I \mathrm{Y} \rightarrow \mathrm{X}}\right)$ is the number of gene copies at locus $l$ in individual $\mathrm{X}(\mathrm{Y})$ for which the allelic state is also observed (that is, shared) in individual Y (X). Calculations of allele-shared distances between pairs of individuals and construction of neighbour-joining dendrograms were performed with the program TreeMaker (S. Piry, personal communication). Dendrograms were constructed using the TreeView 1.6.6 $\operatorname{program}^{27}$.

\section{Received 5 February; accepted 4 May 2005.}

1. Hamilton, W. D. Extraordinary sex ratios. Science 156, 477-488 (1967).

2. Eberhard, W. G. Evolutionary consequences of intracellular organelle competition. Q. Rev. Biol. 55, 231-249 (1980)

3. Hurst, L. D. Intragenomic conflict as an evolutionary force. Proc. R. Soc. Lond. B 248, 135-140 (1992).

4. Pomiankowski, A. in Levels of Selection in Evolution (ed. Keller, L.) 121-152 (Princeton Univ. Press, Princeton, 1999)

5. Hölldobler, B. \& Wilson, E. O. The Ants (Springer, Berlin, 1990).

6. Torres, J. A., Snelling, R. R. \& Canals, M. Seasonal and nocturnal periodicities in ant nuptial flights in the Tropics (Hymenoptera: Formicidae). Sociobiology 37, 601-626 (2001)

7. Sherman, P. W., Seeley, T. D. \& Reeve, H. K. Parasites, pathogens, and polyandry in social Hymenoptera. Am. Nat. 131, 602-610 (1988). 
8. Crozier, R. H. \& Page, R. E. On being the right size: male contributions and multiple mating in social Hymenoptera. Behav. Ecol. Sociobiol. 18, 105-115 (1985).

9. Cole, B. J. \& Wiernasz, D. C. The selective advantage of low relatedness. Science 285, 891-893 (1999).

10. Pearcy, M., Aron, S., Doums, C. \& Keller, L. Conditional use of sex and parthenogenesis for worker and queen production in ants. Science 306, 1780-1783 (2004).

11. Baudry, E. et al. Whole-genome scan in thelytokous-laying workers of the Cape honeybee (Apis mellifera capensis): central fusion, reduced recombination rates and centromere mapping using half-tetrad analysis. Genetics 167, 243-252 (2004).

12. Koeniger, N., Hemmling, C. \& Yoshida, T. Drones as sons of drones in Apis mellifera. Apidologie (Celle) 20, 391-394 (1989).

13. Cook, J. M. \& Crozier, R. H. Sex determination and population biology in the Hymenoptera. Trends Ecol. Evol. 10, 281-286 (1995).

14. Simon, J.-C., Delmotte, F., Rispe, C. \& Crease, T. Phylogenetic relationships between parthenogens and their sexual relatives: the possible routes to parthenogenesis in animals. Biol. J. Linn. Soc. 79, 151-163 (2003).

15. Normark, B. B. The evolution of alternative genetic systems in insects. Annu. Rev. Entomol. 48, 397-423 (2003).

16. Werren, J. H., Nur, U. \& Eickbush, D. An extrachromosomal factor causing loss of paternal chromosomes. Nature 327, 75-76 (1987).

17. Nur, U., Werren, J. H., Eickbush, D. G., Burke, W. D. \& Eickbush, T. H. A 'selfish' $B$ chromosome that enhances its transmission by eliminating the paternal genome. Science 240, 512-514 (1988)

18. Ulloa-Chacon, P. \& Cherix, D. Quelques aspects de la biologie de Wasmannia auropunctata (Roger) (Hymenoptera, Formicidae). Actes Colloq. Insectes Sociaux 4, 177-184 (1988).

19. Rice, W. R. Sexually antagonistic male adaptation triggered by experimental arrest of female evolution. Nature 381, 232-234 (1996).

20. Swanson, W. J., Yang, Z., Wolfner, M. F. \& Aquadro, C. F. Positive Darwinian selection drives the evolution of several female reproductive proteins in mammals. Proc. Natl Acad. Sci. USA 98, 2509-2514 (2001)

21. Arnqvist, G. \& Rowe, L. Antagonistic coevolution between the sexes in a group of insects. Nature 415, 787-789 (2002).

22. Helms-Cahan, S. \& Keller, L. Complex hybrid origin of genetic caste determination in harvester ants. Nature 424, 306-309 (2003).

23. Parker, J. D. A major evolutionary transition to more than two sexes? Trends Ecol. Evol. 19, 83-86 (2004)

24. Chapuisat, M. Mating frequency of ant queens with alternative dispersal strategies, as revealed by microsatellite analysis of sperm. Mol. Ecol. 7, 1097-1105 (1998)

25. Fournier, D. et al. Characterization and PCR multiplexing of polymorphic microsatellite loci for the invasive ant Wasmannia auropunctata. Mol. Ecol. Notes 5, 239-242 (2005).

26. Raymond, M. \& Rousset, F. GENEPOP (version 1.2): population genetics software for exact tests and ecumenicism. J. Hered. 86, 248-249 (1995).

27. Page, R. D. M. TreeView: an application to display phylogenetic trees on personal computers. Comput. Appl. Biosci. 12, 357-358 (1996)

Acknowledgements We are grateful to the Laboratoire Environment de Petit Saut (EDF-CNEH) for hosting us during field work, the Centre de Coopération Internationale en Recherche Agronomique pour le Développement (CIRAD) of French Guiana for allowing access to plantations, S. Aron for performing flow cytometry analysis, and M. Chapuisat, P. Christe, D. Haig, R. Hammond, S. Helms Cahan, K. Parker and D. Queller for comments on the manuscript. The work was supported by grants from the French Ministère de l'Écologie et du Développement Durable to A.E. and the Swiss NSF to L.K.

Author Information Reprints and permissions information is available at npg.nature.com/reprintsandpermissions. The authors declare no competing financial interests. Correspondence and requests for materials should be addressed to D.F. (denis.fournier@ulb.ac.be) or A.E. (estoup@ensam.inra.fr). 